\title{
GENERALIZED FOURIER-STIELTJES ALGEBRA
}

\author{
By
}

\section{G. A. BAGHERI-BARDI}

Abstract. Let $\left\{G_{i}\right\}_{i=1}^{n}$ be locally compact groups and $\mathscr{H}$ be Hilbert space. We define the n-variable Fourier-Stieltjes algebra $B\left(\prod_{1}^{n} G_{i}, \mathbf{B}(\mathscr{H})\right)$ consists all functions

$$
\phi: G_{1} \times \cdots \times G_{n} \rightarrow \mathbf{B}(\mathscr{H})
$$

for which there exists unitary representations $\pi_{i}: \mathbf{G}_{i} \rightarrow \mathbf{B}\left(\mathscr{H}_{i}\right)$ and a diagram of bounded operators

$$
\mathscr{H} \stackrel{V}{\longrightarrow} \mathscr{H}_{n} \stackrel{T_{n-1}}{\longrightarrow} \mathscr{H}_{n-1} \cdots \mathscr{H}_{2} \stackrel{T_{1}}{\longrightarrow} \mathscr{H}_{1} \stackrel{U}{\longrightarrow} \mathscr{H}
$$

with

$$
\phi\left(s_{1}, \ldots, s_{n}\right)=U \pi_{1}\left(s_{1}\right) T_{1} \pi_{2}\left(s_{2}\right) \cdots \pi_{n-1}\left(s_{n-1}\right) T_{n} \pi_{n}\left(s_{n}\right) V
$$

We extend the pointwise product on $B\left(\prod_{1}^{n} G_{i}, \mathbf{B}(\mathscr{H})\right)$ under which it forms a completely contractive commutative unital Banach algebra. A diagram of its subalgebras will be introduced.

\section{Introduction}

Once and for all in this paper $\mathbf{G}_{\mathbf{1}}$ and $\mathbf{G}_{\mathbf{2}}$ are locally compact groups and $\mathscr{H}$ is a Hilbert space with a fixed orthogonal basis $\mathscr{E}$. We denote by $\mathbf{B}(\mathscr{H})(\mathscr{K}(\mathscr{H}))$ the algebra of all bounded (compact) operators on $\mathscr{H}$.

The bi-Fourier-Stieltjes algebra $B^{2}\left(\mathbf{G}_{\mathbf{1}} \times \mathbf{G}_{\mathbf{2}}\right)$ was introduced and studied as a dual operator space in [9] and [16]. It consists all bi-coefficients of unitary representations of $\mathbf{G}_{\mathbf{1}}$ and $\mathbf{G}_{\mathbf{2}}: \phi: \mathbf{G}_{\mathbf{1}} \times \mathbf{G}_{\mathbf{2}} \rightarrow \mathbf{C}$ is in $B^{2}\left(\mathbf{G}_{\mathbf{1}} \times \mathbf{G}_{\mathbf{2}}\right)$ if there exist unitary representations $\pi_{i}: \mathbf{G}_{i} \rightarrow \mathbf{B}\left(\mathscr{H}_{i}\right)$, (connector) bounded operator $T: \mathscr{H}_{2} \rightarrow$

2010 Mathematics Subject Classification: Primary 43A30, 47L25, 46L07.

Key words and phrases: Fourier-Stiltjes algebras, Operator spaces, Operator spaces and completely bounded maps.

Received August 18, 2014.

Revised January 13, 2015. 
$\mathscr{H}_{1}$ and $\zeta \in \mathscr{H}_{1}$ and $\eta \in \mathscr{H}_{2}$ such that

$$
\phi(s, t)=\left\langle\pi_{1}(s) T \pi_{2}(t) \eta \mid \zeta\right\rangle
$$

In this paper we introduce the generalized bi-Fourier-Stieltjes algebra $B^{2}\left(\mathbf{G}_{\mathbf{1}} \times \mathbf{G}_{\mathbf{2}}, \mathbf{B}(\mathscr{H})\right)$ as an extension of the bi-Fourier-Stieltjes algebra. It consists all operator bi-coefficients of unitary representations of $\mathbf{G}_{\mathbf{1}}$ and $\mathbf{G}_{\mathbf{2}}: \phi: \mathbf{G}_{\mathbf{1}} \times \mathbf{G}_{\mathbf{2}} \rightarrow$ $\mathbf{B}(\mathscr{H})$ is in $B^{2}\left(\mathbf{G}_{\mathbf{1}} \times \mathbf{G}_{\mathbf{2}}, \mathbf{B}(\mathscr{H})\right)$ if there exist unitary representations $\pi_{i}: \mathbf{G}_{i} \rightarrow$ $\mathbf{B}\left(\mathscr{H}_{i}\right)$ and a diagram of bounded operators

$$
\mathscr{H} \stackrel{V_{2}}{\longrightarrow} \mathscr{H}_{2} \stackrel{T}{\longrightarrow} \mathscr{H}_{1} \stackrel{V_{1}^{*}}{\longrightarrow} \mathscr{H}
$$

such that

$$
\phi(s, t)=V_{1}^{*} \pi_{1}(s) T \pi_{2}(t) V_{2}
$$

We extend the pointwise product on $B^{2}\left(\mathbf{G}_{\mathbf{1}} \times \mathbf{G}_{\mathbf{2}}, \mathbf{B}(\mathscr{H})\right)$ and prove under which the generalized bi-Fourier-Stieltjes algebra is a completely contractive commutative Banach algebra. We also illustrate it by using of the following operator spaces identification

$$
B^{2}\left(\mathbf{G}_{\mathbf{1}} \times \mathbf{G}_{\mathbf{2}}, \mathbf{B}(\mathscr{H})\right) \simeq\left(B\left(\mathbf{G}_{\mathbf{1}}\right) \stackrel{w^{*} h}{\otimes} B\left(\mathbf{G}_{\mathbf{2}}\right)\right) \bar{\otimes} \mathbf{B}(\mathscr{H})
$$

In fact we calculate the imposed pointwise product on the right hand of above and then obtain a diagram of well-behaved subalgebras. Finally we calculate the maximal ideals space and have a discussion about the operator amenability for some particular of those subalgebras.

\section{Preliminaries}

We define a matrix norm $\left\{\|\cdot\|_{n}\right\}$ on a linear space $V$ to be an assignment of a norm $\|\cdot\|_{n}$ on the matrix space $\mathbf{M}_{n}(V)$ for each $n \in \mathbf{N}$. An operator space is a linear space $V$ together with a matrix norm $\left\{\|\cdot\|_{n}\right\}$ for which

(1) $\left\|\left[\begin{array}{ll}v & 0 \\ 0 & w\end{array}\right]\right\| \|_{m+n}=\max \left\{\|v\|_{n},\|w\|_{m}\right\}$

(2) $\|\alpha v \beta\|_{n} \leq\|\alpha\|\|v\|_{n}\|\beta\|$

for all $v \in \mathbf{M}_{n}(V), w \in \mathbf{M}_{m}(W)$ and $\alpha \in \mathbf{M}_{m, n}, \beta \in \mathbf{M}_{m, n}$. In [17] Ruan proved operator spaces are just the abstract case of subspaces of $C^{*}$-algebras.

Given operator spaces $X$ and $Y$. We also let $C B(X, Y)$ the linear space of completely bounded linear maps, i.e. maps $T: X \rightarrow Y$ for which the amplifi- 
cation, $T_{(n)}: M_{n}(X) \rightarrow M_{n}(Y)$, satisfy

$$
\|T\|_{c b}=\sup \left\{\left\|T_{(n)}\right\|: n \in \mathbf{N}\right\}<+\infty
$$

Also $T$ is called completely isometry (contraction) if $T_{(n)}$ is isometry (contraction) for all $n \in \mathbf{N}$. We will assign $X^{*}=C B(X, \mathbf{C})$ the operator space from [4]. We will make use of tensor product here but just recall those facts which will be used. For definitions and basic properties see the corresponded references.

Similar to classical case the operator projective tensor product linearize completely bounded bilinear maps i.e. (see [3] [12] or [13] section 7)

$$
C B(X \times Y, \mathbf{C}) \simeq C B\left(X, Y^{*}\right)=(X \widehat{\otimes} Y)^{*}
$$

For some particular case like $Y^{*}=\mathbf{B}(\mathscr{H})$ dual of the operator projective tensor product may be expressed as the normal spacial tensor product i.e. (see [2] 1.6.5)

$$
(X \widehat{\otimes} \mathbf{T}(\mathscr{H}))^{*}=C B(X, \mathbf{B}(\mathscr{H}))=X^{*} \bar{\otimes} \mathbf{B}(\mathscr{H})
$$

where $\bar{\otimes}$ is the normal spatial tensor product ([13] or [18] section 7$)$ and $\mathbf{T}(\mathscr{H})$ is the set of all trace class operators on $\mathscr{H}$.

The operator injective tensor product is denoted by $\otimes$ (see [12] [3] or [13] section 8). In general we have

$$
X^{*} \stackrel{\vee}{\otimes} Y \hookrightarrow C B(X, Y)
$$

Therefore

$$
X^{*} \stackrel{\vee}{\otimes} \mathbf{B}(\mathscr{H}) \hookrightarrow X^{*} \bar{\otimes} \mathbf{B}(\mathscr{H})
$$

Finally the Haagerup tensor product ([10] or [13] section 9$)$ is denoted by $\stackrel{h}{~}^{\text {. In }}$ [4] Blecher and Smith characterized the dual of the Haagerup tensor product in terms of what they called the weak*-Haggerup tensor product i.e.

$$
(X \stackrel{h}{\otimes} Y)^{*}=X^{*} \stackrel{w^{*} h}{\otimes} Y^{*}
$$

Also $u$ is in $X^{*} \otimes w^{*} h Y^{*}$ if and only if $u$ has a $w^{*}$-representation $\sum x_{i} \otimes y_{i}$ where $\left[x_{i}\right] \in M_{1, I}\left(X^{*}\right),\left[y_{i}\right]_{I, 1} \in M_{I, 1}\left(Y^{*}\right)$ for some cardinal number $I$ and

$$
\|u\|_{w^{*} h}=\inf \left\{\left\|\left[x_{i}\right]\right\|\left\|\left[y_{i}\right]\right\|:\left[x_{i}\right] \in M_{1, I}\left(X^{*}\right),\left[y_{i}\right]_{I, 1} \in M_{I, 1}\left(Y^{*}\right)\right\}
$$

which is actually achieved [4].

Let $\mathscr{A}$ be an algebra over complex numbers. We call $\mathscr{A}$ a completely contractive Banach algebra if $\mathscr{A}$ is a complete operator space and the multiplication $\mathscr{A} \times \mathscr{A} \rightarrow \mathscr{A}$ is completely contractive, i.e. it extends on the operator projective 
tensor product $\mathscr{A} \widehat{\otimes} \mathscr{A} \rightarrow \mathscr{A}$. Equivalently, we have

$$
\left\|\left[a_{i, j} b_{k, l}\right]\right\| \leq\left\|\left[a_{i, j}\right]\right\|\left\|\left[b_{i, j}\right]\right\|
$$

for all $a=\left[a_{i, j}\right]$ and $b=\left[b_{i, j}\right]$ in $M_{n}(\mathscr{A})$ (See [13] section 17). Completely contractive Banach algebra were first mentioned in [4]; it was observed there that the Fourier algebras of a locally compact group were examples of these.

We fix $\omega$ to be the isometry defined by

$$
\omega: \mathscr{H} \rightarrow \mathscr{H} \otimes{ }^{2} \mathscr{H}: \omega e=e \otimes e \quad(e \in \mathscr{E})
$$

Given $a, b \in B(\mathscr{H})$, we regard $a \otimes b$ as an element of $\mathbf{B}\left(\mathscr{H} \otimes{ }^{2} \mathscr{H}\right)$ and we define the Schur product of $a$ and $b$ as

$$
a * b=\omega^{*}(a \otimes b) \omega
$$

Equipped with the natural operator space and the Schur product, $\mathbf{B}(\mathscr{H})$ and $\mathscr{K}(\mathscr{H})$ are completely contractive commutative Banach algebras (See 5.3.11 from [2]). The main reference for the Schur product is [19].

\section{Generalized Bi-Fourier-Stieltjes Algebra}

Let $\mathscr{X}$ be a locally compact topological space. We denote by $C_{b}(\mathscr{X}, \mathbf{B}(\mathscr{H}))$ the set of all (norm) bounded continuous functions $f: \mathscr{X} \rightarrow \mathbf{B}(\mathscr{H})$. For each $n \in \mathbf{N}$, we have the following natural linear spaces identification

$$
\mathbf{M}_{n}\left(C_{b}(\mathscr{X}, \mathbf{B}(\mathscr{H}))\right) \simeq C_{b}\left(\mathscr{X}, \mathbf{B}\left(\mathscr{H}^{n}\right)\right)
$$

Therefore we may say the uniform norm determines an operator space structure on $C_{b}(\mathscr{X}, \mathbf{B}(\mathscr{H}))$. We extend the pointwise product on $C_{b}(\mathscr{X}, \mathbf{B}(\mathscr{H}))$

$$
f \bullet g(x):=f(x) * g(x)
$$

where $f(x) * g(x)$ is the Schur product of the operators $f(x)$ and $g(x)$ [19].

Proposition 1. Under the point-wise product $C_{b}(\mathscr{X}, \mathbf{B}(\mathscr{H}))$ is a completely contractive commutative Banach algebra.

Proof. Since the Schur product is completely contractive on $\mathbf{B}(\mathscr{H}$ ) (See 5.3.11 from [2]) then the assertion is obtained.

For given $n \in \mathbf{N}$, we say $\Phi: \mathbf{G}_{\mathbf{1}} \times \mathbf{G}_{\mathbf{2}} \rightarrow \mathbf{B}\left(\mathscr{H}^{n}\right)$ is h-bounded if it has an h-Stinespring's representation i.e. there exist unitary representations $\pi_{i}: \mathbf{G}_{i} \rightarrow$ 
$\mathbf{B}\left(\mathscr{H}_{i}\right)$ and a diagram of bounded operators

$$
\mathscr{H} \stackrel{V_{2}}{\longrightarrow} \mathscr{H}_{2} \stackrel{T}{\longrightarrow} \mathscr{H}_{1} \stackrel{V_{1}^{*}}{\longrightarrow} \mathscr{H}
$$

such that

$$
\Phi(s, t)=V^{*} \pi_{1}(s) T \pi_{2}(t) W
$$

We denote $B^{2}\left(\mathbf{G}_{\mathbf{1}} \times \mathbf{G}_{\mathbf{2}}, \mathbf{B}(\mathscr{H})\right)$ by the set of all h-bounded maps $\Phi: \mathbf{G}_{\mathbf{1}} \times \mathbf{G}_{\mathbf{2}} \rightarrow$ $\mathbf{B}(\mathscr{H})$ and call it the generalized bi-Fourier-Stieltjes algebra. Note that for given h-bounded maps $i=1,2$

$$
\Phi_{i}=V_{i}^{*} \pi_{i}(.) T_{i} \sigma_{i}(.) W_{i}
$$

We have

$$
\Phi_{1}+\Phi_{2}=\left[\begin{array}{l}
V_{1} \\
V_{2}
\end{array}\right]^{*}\left[\begin{array}{cc}
\pi_{1} & 0 \\
0 & \pi_{2}
\end{array}\right]\left[\begin{array}{cc}
T_{1} & 0 \\
0 & T_{2}
\end{array}\right]\left[\begin{array}{cc}
\sigma_{1} & 0 \\
0 & \sigma_{2}
\end{array}\right]\left[\begin{array}{l}
W_{1} \\
W_{2}
\end{array}\right]
$$

Therefore $B^{2}\left(\mathbf{G}_{\mathbf{1}} \times \mathbf{G}_{\mathbf{2}}, \mathbf{B}(\mathscr{H})\right)$ is a subspace of $C_{b}\left(\mathbf{G}_{\mathbf{1}} \times \mathbf{G}_{\mathbf{2}}, \mathbf{B}(\mathscr{H})\right)$. It is directly to be checked that

$$
\mathbf{M}_{n}\left(B^{2}\left(\mathbf{G}_{\mathbf{1}} \times \mathbf{G}_{\mathbf{2}}, \mathbf{B}(\mathscr{H})\right)\right) \rightarrow B^{2}\left(\mathbf{G}_{\mathbf{1}} \times \mathbf{G}_{\mathbf{2}}, \mathbf{B}\left(\mathscr{H}^{n}\right)\right):\left[\phi_{i, j}\right] \rightarrow \Phi
$$

is a linear spaces isomorphism, where $\Phi(t, s)=\left[\phi_{i, j}(t, s)\right]$. From now on we identify $\left[\phi_{i, j}\right]$ and $\Phi$.

REMARK 2. It is well-known (see [6] or [13] corollary 9.4.6) for each completely bounded map $\tilde{\phi}$ in $C B\left(C^{*}\left(\mathbf{G}_{\mathbf{1}}\right) \stackrel{h}{\otimes} C^{*}\left(\mathbf{G}_{\mathbf{2}}\right), \mathbf{B}(\mathscr{H})\right)$ there exist nondegenerate *-representations $\tilde{\pi}_{i}$ of $C^{*}\left(\mathbf{G}_{i}\right)$, bounded operator $T: \mathscr{H}_{\tilde{\pi}_{2}} \rightarrow \mathscr{H}_{\tilde{\pi}_{1}}$ and bounded operators $V, W: \mathscr{H} \rightarrow \mathscr{H}_{\tilde{\pi}_{i}}$ such that

$$
\phi=V^{*} \tilde{\pi}_{1} T \tilde{\pi}_{2} W, \quad\|\phi\|_{c b}=\|V\|\|T\|\|W\|
$$

Let $\Phi=\left[\phi_{i, j}\right]: \mathbf{G}_{\mathbf{1}} \times \mathbf{G}_{\mathbf{2}} \rightarrow \mathbf{B}\left(\mathscr{H}^{n}\right)$. We define

$$
\left\|\left[\phi_{i, j}\right]\right\|_{n}:=\inf \|V\|\|T\|\|W\|
$$

where the infimum is taken over all h-Stinespring's representations $\Phi=$ $V^{*} \pi T \sigma W$. The matrix norms $\left\{\|\cdot\|_{n}\right\}_{n=1}^{\infty}$ puts an operator space structure on $B^{2}\left(\mathbf{G}_{\mathbf{1}} \times \mathbf{G}_{\mathbf{2}}, \mathbf{B}(\mathscr{H})\right)$ under which the following map is a completely isometry isomorphism

$$
\begin{gathered}
B^{2}\left(\mathbf{G}_{\mathbf{1}} \times \mathbf{G}_{\mathbf{2}}, \mathbf{B}(\mathscr{H})\right) \rightarrow C B\left(C^{*}\left(\mathbf{G}_{\mathbf{1}}\right) \stackrel{h}{\otimes} C^{*}\left(\mathbf{G}_{\mathbf{2}}\right), \mathbf{B}(\mathscr{H})\right) \\
V^{*} \pi T \sigma W \rightarrow V^{*} \tilde{\pi} T \tilde{\sigma} W
\end{gathered}
$$


where $\tilde{\pi}$ and $\tilde{\sigma}$ are the corresponding non-degenerate $*$-representations of $\pi$ and $\sigma$ on $C^{*}\left(\mathbf{G}_{\mathbf{1}}\right)$ and $C^{*}\left(\mathbf{G}_{\mathbf{2}}\right)$ respectively.

The generalized bi-Fourier-Stieltjes algebra $B^{2}\left(\mathbf{G}_{\mathbf{1}} \times \mathbf{G}_{\mathbf{2}}, \mathbf{B}(\mathscr{H})\right)$ is closed under the pointwise product (see 3.0.7). In other words it is a subalgebra of $C_{b}\left(\mathbf{G}_{\mathbf{1}} \times \mathbf{G}_{\mathbf{2}}, \mathbf{B}(\mathscr{H})\right)$. To prove it we take the h-Stinespring's representations

$$
\phi(s, t)=V_{1}^{*} \pi_{1}(s) T \pi_{2}(t) V_{2}, \quad \psi(s, t)=W_{1}^{*} \sigma_{1}(s) S \sigma_{2}(t) W_{2}
$$

Then

$$
\begin{aligned}
\phi \bullet \psi(s, t) & =\phi(s, t) * \psi(s, t) \\
& =\omega^{*}(\phi(s, t) \otimes \psi(s, t)) \omega \\
& =\omega^{*}\left(V_{1}^{*} \pi_{1}(s) T \pi_{2}(t) V_{2}\right) \otimes\left(W_{1}^{*} \sigma_{1}(s) S \sigma_{2}(t) W_{2}\right) \omega \\
& =\omega^{*}\left(V_{1} \otimes V_{2}\right)^{*}\left(\pi_{1} \odot \pi_{2}\right)(s)(S \otimes T)\left(\sigma_{1} \odot \sigma_{2}\right)(t)\left(W_{1} \otimes W_{2}\right) \omega
\end{aligned}
$$

where

$$
\pi_{1} \odot \pi_{2}: \mathbf{G}_{\mathbf{1}} \rightarrow \mathbf{B}\left(\mathscr{H}_{\pi_{1}} \otimes^{2} \mathscr{H}_{\pi_{2}}\right): s \rightarrow \pi_{1}(s) \otimes \pi_{2}(s)
$$

THEOREM 3. Let $\mathbf{G}_{\mathbf{1}}$ and $\mathbf{G}_{\mathbf{2}}$ be locally compact groups. Then the generalized bi-Fourier-Stieltjes algebra $B^{2}\left(\mathbf{G}_{\mathbf{1}} \times \mathbf{G}_{\mathbf{2}}, \mathbf{B}(\mathscr{H})\right)$ is a completely contractive commutative Banach algebra under the point-wise product.

Proof. Let $\Phi_{1}=\left[\phi_{i, j}\right]_{n \times n}$ and $\Phi_{2}=\left[\psi_{i, j}\right]_{n \times n}$ be h-bounded maps and put

$$
\Phi_{1} \otimes \Phi_{2}=\left[\begin{array}{ccc}
\left(\phi_{1,1} \bullet \psi_{i, j}\right) & \cdots & \left(\phi_{1, n} \bullet \psi_{i, j}\right) \\
\vdots & & \vdots \\
\left(\phi_{n, 1} \bullet \psi_{i, j}\right) & \cdots & \left(\phi_{n, n} \bullet \psi_{i, j}\right)
\end{array}\right]
$$

We take the following h-Stinespring's representations

$$
\Phi_{1}=V^{*} \pi_{1} T \pi_{2} W, \quad \Phi_{2}=M^{*} \sigma_{1} S \sigma_{2} N
$$

With

$$
\left\|\Phi_{1}\right\|=\|V\|\|T\|\|W\|, \quad\left\|\Phi_{2}\right\|=\|M\|\|S\|\|N\|
$$

We consider the projections

$$
P_{i}: \mathscr{H}^{n} \rightarrow \mathscr{H}:\left(h_{1}, \ldots, h_{n}\right) \rightarrow h_{i}
$$

and put $E_{i}=P_{i}^{*}$. Then 


$$
\begin{aligned}
& \left(V^{*} \otimes M^{*}\right)\left(\pi_{1} \otimes \sigma_{1}\right)(T \otimes S)\left(\pi_{2} \otimes \sigma_{2}\right)(W \otimes N)=\left(\left[\begin{array}{l}
P_{1} V^{*} \\
P_{2} V^{*} \\
P_{3} V^{*}
\end{array}\right] \otimes\left[\begin{array}{l}
P_{1} M^{*} \\
P_{2} M^{*} \\
P_{3} M^{*}
\end{array}\right]\right) \\
& \left(\pi_{1} \otimes \sigma_{1}\right)(T \otimes S)\left(\left[\begin{array}{lll}
W E_{1} & W E_{2} & W E_{3}
\end{array}\right] \otimes\left[\begin{array}{lll}
N E_{1} & N E_{2} & N E_{3}
\end{array}\right]\right) \\
& =\left[\left(P_{i} V^{*}\right) \pi_{1} T \sigma_{1}\left(M E_{j}\right)\right]_{n \times n} \otimes\left[\left(P_{k} M^{*}\right) \pi_{2} T \sigma_{2}\left(N E_{l}\right)\right]_{n \times n} \\
& =\Phi_{1} \otimes \Phi_{2}
\end{aligned}
$$

Therefore

$$
\begin{aligned}
\left\|\Phi_{1} \otimes \Phi_{2}\right\| & \leq\left\|\left(V^{*} \otimes M^{*}\right)\left(\pi_{1} \otimes \sigma_{1}\right)(T \otimes S)\left(\pi_{2} \otimes \sigma_{2}\right)(W \otimes N)\right\| \\
& \leq(\|V\|\|T\|\|W\|)(\|M\|\|S\|\|N\|) \\
& \leq\left\|\Phi_{1}\right\|\left\|\Phi_{2}\right\|
\end{aligned}
$$

By definition of the Schur product one may see in particular case $\mathscr{H}=\mathbf{C}$ the pointwise product is given by the natural way.

COROLlary 4. The bi-Fourier-Stieltjes algebra $B^{2}\left(\mathbf{G}_{\mathbf{1}} \times \mathbf{G}_{\mathbf{2}}\right)$ is a unital completely contractive Banach algebra under the pointwise product.

Now we begin to illustrate the generalized bi-Fourier-Stieltjes algebra. On the basis [4], we have

$$
\left(C^{*}\left(\mathbf{G}_{\mathbf{1}}\right) \stackrel{h}{\otimes} C^{*}\left(\mathbf{G}_{\mathbf{2}}\right)\right)^{*}=B\left(\mathbf{G}_{\mathbf{1}}\right) \stackrel{w^{*} h}{\otimes} B\left(\mathbf{G}_{\mathbf{2}}\right)
$$

Therefore there is a completely isometry isomorphism from the bi-FourierStieltjes algebra $B^{2}\left(\mathbf{G}_{\mathbf{1}} \times \mathbf{G}_{\mathbf{2}}\right)$ onto the weak*-Haagerup tensor product of the Fourier-Stieltjes algebras $B\left(\mathbf{G}_{\mathbf{1}}\right)$ and $B\left(\mathbf{G}_{\mathbf{2}}\right)$ which we show by

$$
j: B\left(\mathbf{G}_{\mathbf{1}}\right) \stackrel{w^{*} h}{\otimes} B\left(\mathbf{G}_{\mathbf{2}}\right) \rightarrow B^{2}\left(\mathbf{G}_{\mathbf{1}} \times \mathbf{G}_{\mathbf{2}}\right)
$$

We may also assume $j$ is $w^{*}$-continuous. Therefor we obtain the following dual operator spaces identifications

$$
\begin{aligned}
B^{2}\left(\mathbf{G}_{\mathbf{1}} \times \mathbf{G}_{\mathbf{2}}, \mathbf{B}(\mathscr{H})\right) & =C B\left(C^{*}\left(\mathbf{G}_{\mathbf{1}}\right) \stackrel{h}{\otimes} C^{*}\left(\mathbf{G}_{\mathbf{2}}\right), \mathbf{B}(\mathscr{H})\right) \\
& =\left(\left(C^{*}\left(\mathbf{G}_{\mathbf{1}}\right) \stackrel{h}{\otimes} C^{*}\left(\mathbf{G}_{\mathbf{2}}\right)\right) \widehat{\otimes} \mathbf{T}(\mathscr{H})\right)^{*}
\end{aligned}
$$




$$
\begin{aligned}
& =\left(B\left(\mathbf{G}_{\mathbf{1}}\right) \stackrel{w^{*} h}{\otimes} B\left(\mathbf{G}_{\mathbf{2}}\right)\right) \bar{\otimes} \mathbf{B}(\mathscr{H}) \\
& =B^{2}\left(\mathbf{G}_{\mathbf{1}} \times \mathbf{G}_{\mathbf{2}}\right) \bar{\otimes} \mathbf{B}(\mathscr{H})
\end{aligned}
$$

We partially calculate the imposed point-wise product on its tensor type.

One may conclude by the beginning part of section 7 of [11]

$$
\left(f_{1} \otimes g_{1}\right) \cdot\left(f_{2} \otimes g_{2}\right):=f_{1} f_{2} \otimes g_{1} g_{2}
$$

induces a completely contractive multiplication on $B\left(\mathbf{G}_{\mathbf{1}}\right) \stackrel{w^{*} h}{\otimes} B\left(\mathbf{G}_{\mathbf{2}}\right)$. First we show that the bi-Fourier-Stieltjes algebra $B^{2}\left(\mathbf{G}_{\mathbf{1}} \times \mathbf{G}_{\mathbf{2}}\right)$ and $B\left(\mathbf{G}_{\mathbf{1}}\right) \otimes B\left(\mathbf{G}_{\mathbf{2}}\right)$ are the same in the category of completely contractive Banach algebras which will be done in the following three steps.

Step 1. In this step for given $w^{*}$-representation

$$
u=\sum \phi_{i} \otimes \psi_{i} \in B\left(\mathbf{G}_{\mathbf{1}}\right) \stackrel{w^{*} h}{\otimes} B\left(\mathbf{G}_{\mathbf{2}}\right)
$$

we give a suitable h-Stinespring's representation for $j(u)$ (see 3.0.9).

Let the basic element $\phi \otimes \psi$ in $B\left(\mathbf{G}_{\mathbf{1}}\right) \otimes B\left(\mathbf{G}_{\mathbf{2}}\right)$. Then

$$
j(\phi \otimes \psi)=\left\langle\pi_{1}(\zeta \odot k) \sigma_{1} h \mid \eta\right\rangle
$$

where

$$
\phi(x)=\left\langle\pi_{1}(x) \zeta \mid \eta\right\rangle, \quad \psi(y)=\left\langle\sigma_{1}(y) h \mid k\right\rangle
$$

and

$$
\zeta \odot k: \mathscr{H}_{\sigma} \rightarrow \mathscr{H}_{\pi}: h_{1} \rightarrow\left\langle h_{1} \mid k\right\rangle \zeta
$$

As in [4] we may assume

$$
\left\|\sum \phi_{i} \otimes \psi_{i}\right\|_{w^{*} h}=\left\|\left[\phi_{i}\right]_{1, I}\right\|\left\|\left[\psi_{i}\right]_{I, 1}\right\|
$$

where $\left[\phi_{i}\right] \in M_{1, I}\left(B\left(\mathbf{G}_{\mathbf{1}}\right)\right)$ and $\left[\psi_{i}\right] \in M_{I, 1}\left(B\left(\mathbf{G}_{\mathbf{2}}\right)\right)$. By the generalized Stinespring's theorem ([14] or [15] theorem 8.4) there is a continuous unitary representation $\pi_{u}$, bounded operator $T_{u}: l^{2}(I) \rightarrow \mathscr{H}_{\pi}$ and $\eta_{u} \in \mathscr{H}_{\pi}$ with

$$
\left[\phi_{i}\right]_{1, I}=\eta_{u}^{*} \pi_{u} T_{u}, \quad\left\|\left[\phi_{i}\right]_{1, I}\right\|=\left\|\eta_{u}\right\|\left\|T_{u}\right\|
$$

Similarly there is a continuous unitary representation $\sigma_{u}$, bounded operator $V_{u}: l^{2}(I) \rightarrow \mathscr{H}_{\sigma}$ and $h_{u} \in \mathscr{H}_{\sigma}$ with

$$
\left[\psi_{i}\right]_{I, 1}=V_{u}^{*} \sigma_{u} h_{u}, \quad\left\|\left[\psi_{i}\right]_{I, 1}\right\|=\left\|h_{u}\right\|\left\|V_{u}\right\|
$$


We put $T_{u}\left(e_{i}\right)=\zeta_{i}$ and $V_{u}\left(e_{i}\right)=k_{i}$ where $\left\{e_{i}\right\}$ is the standard orthogonal basis of $l^{2}(I)$. Since $\left[\phi_{i}\right]_{1, I}$ and $\left[\psi_{i}\right]_{I, 1}$ are row and column matrices respectively then

$$
\phi_{i}=\left\langle\pi_{u} \zeta_{i}, \eta_{u}\right\rangle, \quad \psi_{i}=\left\langle\sigma_{u} h_{u}, k_{i}\right\rangle
$$

We put for each finite subset $\mathscr{F} \subseteq I$

$$
\begin{aligned}
T_{\mathscr{F}}=\left\langle\pi_{u}\left(\sum_{\mathscr{F}} \zeta_{i} \odot k_{i}\right) \sigma_{u} h_{u} \mid \eta_{u}\right\rangle & =\left\langle\pi_{u}\left(\sum_{\mathscr{F}} T_{u}\left(e_{i}\right) \odot V_{u}\left(e_{i}\right)\right) \sigma_{u} h_{u} \mid \eta_{u}\right\rangle \\
& =\left\langle\pi_{u}\left(T_{u}\left(\sum_{\mathscr{F}} e_{i} \odot e_{i}\right) V_{u}^{*}\right) \sigma_{u} h_{u} \mid \eta_{u}\right\rangle
\end{aligned}
$$

Therefore $\left\{T_{\mathscr{F}}\right\}_{\mathscr{F} \subseteq I}$ is a uniformly bounded net and for each finite subset $\mathscr{F} \subseteq I$, $T_{\mathscr{F}}$ is a corresponded h-Stinespring's representation for $\sum_{\mathscr{F}} \phi_{i} \otimes \psi_{i}$ and so is $w^{*}$ convergent (see 3.0.9). Now we show $T_{\mathscr{F}}$ is $w^{*}$-convergent to $\left\langle\pi_{u} T_{u} V_{u}^{*} \sigma_{u} h_{u} \mid \eta_{u}\right\rangle$. To prove it first we need to show the bounded net of finite ranks operators $\left\{\sum_{\mathscr{F}}\left(\zeta_{i} \odot k_{i}\right)\right\}$ converges to $T_{u} V_{u}^{*}$ in the strong operator topology. Let $h_{\sigma} \in \mathscr{H}_{\sigma}$. Then

$$
\begin{aligned}
T_{u} V_{u}^{*} h_{\sigma} & =T_{u}\left(\sum\left\langle V_{u}^{*} h_{\sigma} \mid e_{i}\right\rangle e_{i}\right) \\
& \left.=\sum T_{u}\left(\left\langle h_{\sigma} \mid V_{u}\left(e_{i}\right)\right\rangle\right) e_{i}\right) \\
& =\sum\left\langle h_{\sigma} \mid V_{u}\left(e_{i}\right)\right\rangle T_{u}\left(e_{i}\right) \\
& =\sum\left(T_{u}\left(e_{i}\right) \odot V_{u}\left(e_{i}\right)\right) h_{\sigma}=\sum\left(\zeta_{i} \odot k_{i}\right) h_{\sigma}
\end{aligned}
$$

Let the basic element $f \otimes g \in C^{*}\left(\mathbf{G}_{\mathbf{1}}\right) \otimes C^{*}\left(\mathbf{G}_{\mathbf{2}}\right)$. We may assume $f, g$ are continuous functions with compact support.

$$
\begin{aligned}
& \left\langle\left\langle\pi_{u} T_{u} V_{u}^{*} \sigma_{u} h_{u} \mid \eta_{u}\right\rangle, f \otimes g\right\rangle \\
& =\iint\left\langle\pi_{u}(x) T_{u} V_{u}^{*} \sigma_{u}(y) h_{u} \mid \eta_{u}\right\rangle f(x) g(y) d x d y \\
& \quad=\iint\left\langle T_{u} V_{u}^{*} \sigma_{u}(y) h_{u} \mid \pi_{u}\left(x^{-1}\right) \eta_{u}\right\rangle f(x) g(y) d x d y \\
& =\lim \iint\left\langle\left(\sum_{\mathscr{F}} \zeta_{i} \odot k_{i}\right) \sigma_{u}(y) h_{u} \mid \pi_{u}\left(x^{-1}\right) \eta_{u}\right\rangle f(x) g(y) d x d y
\end{aligned}
$$




$$
\begin{aligned}
& =\lim \left\langle\left\langle\pi_{u}\left(\sum_{\mathscr{F}} \zeta_{i} \odot k_{i}\right) \sigma_{u} h_{u} \mid \eta_{u}\right\rangle, f \otimes g\right\rangle \\
& =\lim \left\langle T_{\mathscr{F}}, f \otimes g\right\rangle
\end{aligned}
$$

Therefore

$$
j\left(u=\sum \phi_{i} \otimes \psi_{i}\right)=\left\langle\pi_{u} T_{u} V_{u}^{*} \sigma_{u} h_{u} \mid \eta_{u}\right\rangle
$$

Indeed

$$
\left\|\sum \phi_{i} \otimes \psi_{i}\right\|_{h}=\left\|\eta_{u}\right\|\left\|h_{u}\right\|\left\|V_{u}\right\|\left\|T_{u}\right\|
$$

Step 2. Let the basic element $\phi \otimes \psi$ and $w^{*}$-representation $\sum \phi_{i} \otimes \psi_{i}$. In this Step we show

$$
j\left(\left(\sum \phi_{i} \otimes \psi_{i}\right)(\phi \otimes \psi)\right)=j\left(\sum \phi_{i} \otimes \psi_{i}\right) j(\phi \otimes \psi)
$$

Just as in (3.0.13) and (3.0.14) we take the h-Stinespring's representations

$$
j(\phi \otimes \psi)=\left\langle\pi_{1}(\zeta \odot k) \sigma_{1} h, \eta\right\rangle, \quad j\left(\sum \phi_{i} \otimes \psi_{i}\right)=\left\langle\pi_{u} T_{u} V_{u}^{*} \sigma_{u} h_{u} \mid \eta_{u}\right\rangle
$$

Since the multiplication on the Fourier-Stieltjes algebra $B\left(\mathbf{G}_{i}\right)$ is $w^{*}$-separately continuous (i.e. it is a dual completely contractive Banach algebra) then

$$
\left(\sum \phi_{i} \otimes \psi_{i}\right)(\phi \otimes \psi)=\sum \phi_{i} \phi \otimes \psi_{i} \psi
$$

It is obvious that the bounded net $\left\{\left(\sum_{\mathscr{F}} \zeta_{i} \odot k_{i}\right) \otimes(\zeta \odot k)\right\}_{\mathscr{F}}$ is convergent to $T V^{*} \otimes(\zeta \odot k)$ in the strong operator topology. We also note that for each finite subset $\mathscr{F} \subseteq I$

$$
\begin{aligned}
& j\left(\sum_{\mathscr{F}} \phi_{i} \phi \otimes \psi_{i} \psi\right) \\
& \quad=\left\langle\left(\pi_{u} \odot \pi_{1}\right)\left(\left(\sum_{\mathscr{F}} \zeta_{i} \odot k_{i}\right) \otimes(\zeta \odot k)\right)\left(\sigma_{u} \odot \sigma_{1}\right) h_{u} \otimes h \mid \eta_{u} \otimes \eta\right\rangle
\end{aligned}
$$

Therefore similar to process in step 1

$$
j\left(\sum \phi_{i} \phi \otimes \psi_{i} \psi\right)=\left\langle\left(\pi_{u} \odot \pi_{1}\right)\left(\left(T V^{*}\right) \otimes(\zeta \odot k)\right)\left(\sigma_{u} \odot \sigma_{1}\right) h_{u} \otimes h \mid \eta_{u} \otimes \eta\right\rangle
$$


Step 3. Since $j$ is $w^{*}$-continuous in the second variable then one conclude the completely isometry $j$ is also a homomorphism by using of similar method which applied in step 2 .

THEOREM 5. Let $\mathbf{G}_{\mathbf{1}}$ and $\mathbf{G}_{\mathbf{2}}$ be locally compact groups. There is a completely isometry isomomorphism form $B\left(\mathbf{G}_{\mathbf{1}}\right) \otimes B\left(\mathbf{G}_{\mathbf{2}}\right)$ onto $B^{2}\left(\mathbf{G}_{\mathbf{1}} \times \mathbf{G}_{\mathbf{2}}\right)$.

Let $\phi \otimes v$ be a basic element in $B^{2}\left(\mathbf{G}_{\mathbf{1}} \times \mathbf{G}_{\mathbf{2}}\right) \otimes \mathbf{B}(\mathscr{H})$ and the Stinespring's representation $\phi=\langle\pi T \sigma \zeta \mid \eta\rangle$. We define

$$
\begin{gathered}
T_{\eta}: \mathscr{H} \rightarrow H_{\pi} \otimes \mathscr{H}: k \rightarrow \eta \otimes k \\
S_{\zeta, v}: \mathscr{H} \rightarrow H_{\pi} \otimes \mathscr{H}: h \rightarrow \zeta \otimes v h
\end{gathered}
$$

Then we obtain a suitable corresponding Stinespring's representation for $\phi \otimes v$ as follows

$$
\phi \otimes v=T_{\eta}^{*}(\pi \odot \imath)(T \otimes i d)(\sigma \odot \imath) S_{\zeta, v}
$$

Where $l$ is the trivial continuous unitary representation. If we consider the basic elements $\phi_{i} \otimes v_{i}$ in $B^{2}\left(\mathbf{G}_{\mathbf{1}} \times \mathbf{G}_{\mathbf{2}}\right) \otimes \mathbf{B}(\mathscr{H})(i=1,2)$ with the Stinespring's representations

$$
\phi_{i} \otimes v_{i}=T_{\eta_{i}}^{*}\left(\pi_{i} \odot \imath\right)\left(T_{i} \otimes i d\right)\left(\sigma_{i} \odot \imath\right) S_{\zeta_{i}, v_{i}} .
$$

Then for each $e, f$ in $\mathscr{E}$

$$
\begin{aligned}
\left\langle\left(\phi_{1} \otimes\right.\right. & \left.v_{1}\right) \bullet\left(\phi_{2} \otimes v_{2}\right)(x) e|f\rangle \\
= & \left\langle T_{\eta_{1}}^{*}\left(\pi_{1} \odot \imath\right) S_{\zeta_{1} v_{1}} \bullet T_{\eta_{2}}^{*}\left(\pi_{2} \odot \imath\right) S_{\zeta_{2}, v_{2}}(x) e \mid f\right\rangle \\
= & \left\langle\left(T_{\eta_{1}} \otimes T_{\eta_{2}} \omega\right)^{*}\left(\left(\pi_{1} \odot \imath\right) \odot\left(\pi_{2} \odot \imath\right)\right)(x)\left(S_{\zeta_{1}, v_{1}} \otimes S_{\zeta_{2}, v_{2}} \omega\right) e \mid f\right\rangle \\
= & \left\langle\left(\left(\pi_{1}(x) \otimes \imath(x)\right) \otimes\left(\pi_{2}(x) \otimes l(x)\right)\right)\left(\zeta_{1} \otimes v_{1} e\right)\right. \\
& \otimes\left(\zeta_{2} \otimes v_{2} e\right)\left|\left(\eta_{1} \otimes f\right) \otimes\left(\eta_{2} \otimes f\right)\right\rangle \\
= & \left\langle\pi_{1}(x) \zeta_{1} \mid \eta_{1}\right\rangle\left\langle\pi_{2}(x) \zeta_{1} \mid \eta_{1}\right\rangle\left\langle v_{1} e \mid f\right\rangle\left\langle v_{2} e \mid f\right\rangle \\
= & \phi_{1}(x) \phi_{2}(x)\left\langle\omega^{*}\left(v_{1} \otimes v_{2}\right) \omega e \mid f\right\rangle \\
= & \phi_{1}(x) \phi_{2}(x)\left\langle v_{1} * v_{2} e \mid f\right\rangle
\end{aligned}
$$

These calculations show that

$$
\left(\phi_{1} \otimes v_{1}\right) \bullet\left(\phi_{2} \otimes v_{1}\right)=\phi_{1} \phi_{2} \otimes v_{1} * v_{2}
$$


Since the pointwise product is norm continuous on $B^{2}\left(\mathbf{G}_{\mathbf{1}} \times \mathbf{G}_{\mathbf{2}}, \mathbf{B}(\mathscr{H})\right)$ then we have for given uniformly convergence series $\sum_{i=1}^{\infty} \phi_{i} \otimes u_{i}$ and $\sum_{j=1}^{\infty} \psi_{j} \otimes w_{j}$ in $B^{2}\left(\mathbf{G}_{\mathbf{1}} \times \mathbf{G}_{\mathbf{2}}\right) \bar{\otimes} \mathbf{B}(\mathscr{H})$

$$
\left(\sum_{i=1}^{\infty} \phi_{i} \otimes u_{i}\right) \bullet\left(\sum_{j=1}^{\infty} \psi_{j} \otimes w_{j}\right)=\sum_{i, j=1}^{\infty} \phi_{i} \psi_{j} \otimes u_{i} * w_{j}
$$

As a result of the self-duality for the Haagerup tensor product ([8][5][4])

$$
B\left(\mathbf{G}_{\mathbf{1}}\right) \stackrel{h}{\otimes} B\left(\mathbf{G}_{\mathbf{2}}\right) \subseteq B\left(\mathbf{G}_{\mathbf{1}}\right) \stackrel{w^{*} h}{\otimes} B\left(\mathbf{G}_{\mathbf{2}}\right)
$$

Also (see 2.0.4)

$$
B^{2}\left(\mathbf{G}_{\mathbf{1}} \times \mathbf{G}_{\mathbf{2}}\right) \stackrel{\vee}{\otimes} \mathbf{B}(\mathscr{H}) \subseteq B^{2}\left(\mathbf{G}_{\mathbf{1}} \times \mathbf{G}_{\mathbf{2}}\right) \bar{\otimes} \mathbf{B}(\mathscr{H})
$$

We use of the injectivity property of the Haagerup and operator injective tensor product we obtain the following diagram of subalgebras of the generalized Fourier-Stieltjes algebra $B^{2}\left(\mathbf{G}_{\mathbf{1}} \times \mathbf{G}_{\mathbf{2}}, \mathbf{B}(\mathscr{H})\right)$.

$$
\begin{gathered}
\left(B\left(\mathbf{G}_{\mathbf{1}}\right) \stackrel{w^{*} h}{\otimes} B\left(\mathbf{G}_{\mathbf{2}}\right)\right) \bar{\otimes} \mathbf{B}(\mathscr{H}) \\
\left(B\left(\mathbf{G}_{\mathbf{1}}\right) \stackrel{h}{\otimes} B\left(\mathbf{G}_{\mathbf{2}}\right)\right) \stackrel{\vee}{\otimes} \mathscr{K}(\mathscr{H}) \\
\left(A\left(\mathbf{G}_{\mathbf{1}}\right) \stackrel{h}{\otimes} B\left(\mathbf{G}_{\mathbf{2}}\right)+B\left(\mathbf{G}_{\mathbf{1}}\right) \stackrel{h}{\otimes} A\left(\mathbf{G}_{\mathbf{2}}\right)\right) \stackrel{\vee}{\otimes} \mathscr{K}(\mathscr{H}) \\
\left(A\left(\mathbf{G}_{\mathbf{1}}\right) \stackrel{h}{\otimes} B\left(\mathbf{G}_{\mathbf{2}}\right)\right) \stackrel{\vee}{\otimes} \mathscr{K}(\mathscr{H}) \\
\left.\left(A\left(\mathbf{G}_{\mathbf{1}}\right) \stackrel{h}{\otimes} A\left(\mathbf{G}_{\mathbf{2}}\right)\right) \stackrel{\vee}{\otimes} \mathscr{K}\left(\mathbf{G}_{\mathbf{1}}\right) \stackrel{h}{\otimes} A\left(\mathbf{G}_{\mathbf{2}}\right)\right) \stackrel{\vee}{\otimes} \mathscr{K}(\mathscr{H}) \\
\mid \\
0
\end{gathered}
$$

It is well-known that

$$
A\left(\mathbf{G}_{\mathbf{1}}\right) \widehat{\otimes} A\left(\mathbf{G}_{\mathbf{2}}\right) \simeq A\left(\mathbf{G}_{\mathbf{1}} \times \mathbf{G}_{\mathbf{2}}\right)
$$

Since $\|\cdot\|_{h} \leq\|\cdot\|_{\wedge}([2]$ page 36) then we may transfer some properties from the Fourier algebra $A\left(\mathbf{G}_{\mathbf{1}} \times \mathbf{G}_{\mathbf{2}}\right)$ to bi-Fourier algebra $A\left(\mathbf{G}_{\mathbf{1}}\right) \otimes A\left(\mathbf{G}_{\mathbf{2}}\right)$ by the following inclusion mapping

$$
A\left(\mathbf{G}_{\mathbf{1}}\right) \widehat{\otimes} A\left(\mathbf{G}_{\mathbf{2}}\right) \rightarrow A\left(\mathbf{G}_{\mathbf{1}}\right) \stackrel{h}{\otimes} A\left(\mathbf{G}_{\mathbf{2}}\right)
$$


COROLlary 6. (1) The maximal ideal space of the bi-Fourier algebra $A\left(\mathbf{G}_{\mathbf{1}}\right) \otimes A\left(\mathbf{G}_{\mathbf{2}}\right)$ is the cartesian product $\mathbf{G}_{\mathbf{1}} \times \mathbf{G}_{\mathbf{2}}$ and so is semisimple.

(2) The bi-Fourier algebra $A\left(\mathbf{G}_{\mathbf{1}}\right) \circledast A\left(\mathbf{G}_{\mathbf{2}}\right)$ is always weakly amenable.

(3) The bi-Fourier algebra $A\left(\mathbf{G}_{\mathbf{1}}\right) \stackrel{h}{\otimes} A\left(\mathbf{G}_{\mathbf{2}}\right)$ is operator amenable if $\mathbf{G}_{i}$ are amenable.

Proposition 7. Let $\mathbf{G}_{\mathbf{1}}$ and $\mathbf{G}_{\mathbf{2}}$ be compact groups. Then $\mathbf{M}$ is a $w^{*}$-closed maximal ideal of $B^{2}\left(\mathbf{G}_{\mathbf{1}} \times \mathbf{G}_{\mathbf{2}}\right)$ if and only if there is $(x, y) \in \mathbf{G}_{\mathbf{1}} \times \mathbf{G}_{\mathbf{2}}$ such that

$$
\mathbf{M}=\{\langle\pi T \sigma \zeta, \eta\rangle:\langle\pi(x) T \sigma(y) \zeta, \eta\rangle=0\}
$$

Therfore the bi-Fourier-Stieltjes algebra $B^{2}\left(\mathbf{G}_{\mathbf{1}} \times \mathbf{G}_{\mathbf{2}}\right)$ is semisimple.

Proof. Let $\chi$ be a $w^{*}$-continuous character on $B^{2}\left(\mathbf{G}_{\mathbf{1}} \times \mathbf{G}_{\mathbf{2}}\right)$. Then the restriction of $\chi$ is a non-zero character on $A\left(\mathbf{G}_{\mathbf{1}}\right) \otimes A\left(\mathbf{G}_{\mathbf{2}}\right)$ and so it is in the form of $\chi_{1} \otimes \chi_{2}$ where $\chi_{i}$ is a non-zero character of $A\left(\mathbf{G}_{i}\right)$. Since the spectrum of the fourier algebras $A\left(\mathbf{G}_{i}\right)$ are just $\mathbf{G}_{i}$ then there is a $(x, y) \in \mathbf{G}_{\mathbf{1}} \times \mathbf{G}_{\mathbf{2}}$ such that $\chi=$ $\lambda(x) \otimes \lambda(y)$.

\section{References}

[1] G. A. Bagheri-Bardi, A. R. Medghalchi, N. Spronk, Operator valued convolution algebras. Houston J. Math. 36 (4) (2010) 1023-1036.

[2] D. P. Blecher and C. Le Merdy. Operator Algebras and Their Modules, Volume 30 of London Math. Soc., New series. Clarendon Press, Oxford Univ. Press, New York, 2004.

[ 3 ] D. P. Blecher and V. Paulsen. Tensor product of operator spaces. J. Funct. Anal. 99 (1991) 262-92.

[4] D. P. Blecher and R. R. Smith. The dual of the Haagerup tensor product. J. London Math. Soc., 45 (2) (1992) 126-144.

[ 5 ] D. P. Blecher and R. R. Smith. Tensor product of operator spaces II. Canad. J. math. 44 (1992) 75-90.

[6] E. Christensen and A. Sinclair, Representation of completely bounded multilinear operators. J. Funct. Anal. 72 (1987) 151-181.

[ 7 ] P. Eymard, L'algèbre de Fourier d'un group localement compact, Bull. Soc. Math. Franc 92 (1964) 181-236.

[ 8 ] E. G. Effros and J.-Z. Ruan, Self duality for the Haggerup tensor product and Hilbert space factorization.

[9] E. G. Effros and J.-Z. Ruan, Multivariable multipliers for groups and their operator algebras, Proc. Sympos. Pure Math. 511990 part1. 197-218.

[10] E. G. Effros and Z.-J. Ruan. Operator spaces, Volume 23 of London Math. Soc., New Series. Clarendon Press, Oxford Univ. Press, New York, 2000.

[11] E. G. Effros and J.-Z. Ruan, Operator space tensor products and Hopf convolution algebras, J. Operator Theory 50 (2003) 131-156.

[12] E. G. Effros and Z.-J. Ruan. A new approach to operator spaces. Canad. Math. Bull. 34 (1991) 329-37. 
[13] E. G. Effros and Z.-J. Ruan. Operator spaces, Volume 23 of London Math. Soc., New Series. Clarendon Press, Oxford Univ. Press, New York, 2000.

[14] V. I. Paulsen. Every completely polynomially bounded operator is similar to a contraction, J. Funct. Anal. 55 (1984) 225-228.

[15] V. I. Pualsen. Completely Bounded Maps and Operator Algebras, Cambridge University Press, 2002.

[16] Z. J. Ruan. The structure of pure completely bounded and completely positive multilinear operators. Pacific J. of Math. 143 No. 1 (1990) 155-173.

[17] Z. J. Ruan. Subspaces of a $C^{*}$-algebras. J. Funct. Anal., 76 (1988) 217-30.

[18] Z.-J. Ruan. On the predual of dual algebras. J. Operator theory, 27 (1993) 179-92.

[19] Q. F. Stout, Schur multiplication on $\mathbf{B}\left(l_{p}, l_{q}\right)$. J. Operator theory. 5 (1981) 231-243.

\section{Department of Mathematics}

Persian Gulf University

Boushehr 75168, Iran

E-mail: bagheri@mailpgu.ac.ir and alihoular@gmail.com 